\title{
Sistema de costos ambientales para la toma de decisiones en la industria
} bananera

\section{Environmental cost system for decision-making in the banana industry}

\author{
Ingrid Anabel Arámbulo-Carrión \\ ingrid.arambulo37@est.ucacue.edu.ec \\ Universidad Católica de Cuenca, Cuenca \\ Ecuador \\ https://orcid.org/0000-0002-5143-3610 \\ Jose Alciviades Guzmán-Ávila \\ jguzman@ucacue.edu.ec \\ Universidad Católica de Cuenca, Cuenca \\ Ecuador \\ https://orcid.org/0000-0002-1558-8826 \\ Verónica Paulina Moreno-Narváez \\ veronica.moreno@ucacue.edu.ec \\ Universidad Católica de Cuenca, Cuenca \\ Ecuador \\ https://orcid.org/0000-0003-1517-6124
}

Recibido: 05 de agosto de 2020

Aprobado: 01 de octubre de 2020 


\title{
RESUMEN
}

Los costos de producción agrícola tienen características que intervienen en cada proceso y dependen del tipo de cultivo de cada ciclo; el perfeccionamiento de los procedimientos de cultivo permite programar los costos ambientales de manera eficaz, tomando en consideración sucesos imprevistos en escenarios de riesgo, por consiguiente, el objetivo de la investigación es establecer el tratamiento contable de los costos ambientales para los productores bananeros del cantón Machala. La metodología empleada fue de tipo no experimental descriptiva de corte transversal, con el fin de valorar el tipo de costos vinculados al medioambiente; por tanto, los resultados evidenciaron que en la mayoría de Pymes del sector, no se aplican técnicas de cuidado medioambiental y quienes lo hacen no emplean un tratamiento contable adecuado de los costos ambientales, es así que, surge la propuesta de realizar un plan de cuentas y la contabilización respectiva de las transacciones ambientales.

Descriptores: Sistema; decisión; costes; impacto ambiental; competencia. (Palabras tomadas del tesauro UNESCO).

\begin{abstract}
The costs of agricultural production have characteristics that intervene in each process and depend on the type of crop in each cycle; The improvement of the cultivation procedures allows programming the environmental costs in an efficient way, taking into account unforeseen events in risk scenarios, therefore, the objective of the research is to establish the accounting treatment of the environmental costs for the banana producers of the Machala canton. . The methodology used was of a non-experimental descriptive cross-sectional type, in order to assess the type of costs related to the environment; Therefore, the results showed that in the majority of SMEs in the sector, environmental care techniques are not applied and those who do do not use an adequate accounting treatment of environmental costs, thus, the proposal arises to make a chart of accounts and the respective accounting of environmental transactions.
\end{abstract}

Descriptors: System; decision; costs; environmental impact; competence. (Words taken from the UNESCO thesaurus). 
Ingrid Anabel Arámbulo-Carrión; Jose Alciviades Guzmán-Ávila; Verónica Paulina Moreno-Narváez

\section{INTRODUCCIÓN}

La producción bananera y la sistematización de los costos ambientales desde una visión internacional, tiene una connotación directa con la aplicación de las Normas Internacionales de Información Financiera (NIIF) para PYMES, en la que hace referencia al tratamiento de los activos biológicos expresadas en las Normas Internacionales de Contabilidad (NIC), de forma particular en la NIC 41 que direcciona la contabilización de los costos de activos biológicos, que según la NIIF, son los animales vivos y las plantas que desde su siembra forman parte de la empresa, los que deben evolucionar hasta ofrecer productos que se puedan contabilizar, por consiguiente, los activos biológicos expresados en esta norma deben contabilizarse y tener correlación con las actividades agrícolas; sin embargo, la norma no aplica en la transformación y procesamiento de los activos biológicos, a pesar de que tengan semejanza en el dinamismo agrícola.

En este contexto, la producción bananera en la provincia de El Oro tiene sus ventajas al ubicarse en una zona costera que posee un clima apropiado, suelos fértiles y personal calificado para el procesamiento de la fruta, es así que, dichas particularidades benefician al cultivo, productividad y comercialización del banano a países extranjeros, considerándose la principal actividad productiva de la provincia y el país, de igual manera, las mencionadas cualidades se ven reflejadas en la exportación hacia países europeos y asiáticos por ser territorios con alto grado de consumo. Bajo esta situación, nace la necesidad de sistematizar los costos incurridos no solo en la parte convencional, sino también, en los costos ambientales que favorecen a la conservación del medio ambiente. La problemática en la producción bananera en la provincia de El Oro y el Ecuador, se presenta por la utilización sin medida de agroquímicos que están afectando el medioambiente a la par de la deficiente clasificación contable de cada uno de los costos que se incurre para mitigar los impactos del ecosistema, por tanto, la falta de conocimiento de las NIIF y las NIC en la contabilización de los costos ambientales, motiva 
Ingrid Anabel Arámbulo-Carrión; Jose Alciviades Guzmán-Ávila; Verónica Paulina Moreno-Narváez

a realizar acciones sin fundamento, enviando gran parte de los costos a la cuenta contable costos de producción.

Ante lo expuesto, el objetivo de la presente investigación está enfocado en establecer el tratamiento contable de los costos ambientales y su presentación en los estados financieros para que los productores bananeros del cantón Machala tomen decisiones de forma acertada.

\section{Referencial teórico}

Relación entre las Normas Internacionales de Información Financiera para PYMES y la Contabilidad Ambiental

El objetivo de las NIIF está direccionado a su aplicación en los estados financieros y en otros tipos de información financiera en todas las entidades en especial a las pequeñas y medianas empresas conocidas como (PYMES), aplicables a entidades privadas y las que tienen obligación pública de rendir cuentas. Las Normas Internacionales de Información Financiera para Pequeñas y Medianas Entidades (NIIF para las PYMES), se basan en las NIIF completas, con reformas que expresan las necesidades de los interesados de los estados financieros, considerando además el costo-beneficio.

El acrónimo Pymes lo utilizan las pequeñas y medianas empresas, que difiere de sus siglas de cortos espacios de un país con otro; lo cual coincide con el autor (Ron \& Sacoto, 2017) donde expresa que si bien refleja los mismos elementos, en este influye mucho el estatus de desarrollo de cada nación para indicar igualdad o desigualdad. Otros autores como (Yance, Solís, Burgos, \& Hermida, 2017) expresan que el progreso de un país depende mucho de las Pymes lo que da origen a reforzar su ejecución y solicitar estrategias que beneficien a sus operaciones.

El término pequeñas y medianas entidades se basa en la sección 1 de las NIIF definidas por la Junta de Normas Internacionales de Contabilidad (International Accounting Standards Board-IASB), sin embargo, existen muchos sectores en todas partes del 
Ingrid Anabel Arámbulo-Carrión; Jose Alciviades Guzmán-Ávila; Verónica Paulina Moreno-Narváez

mundo que han desarrollado sus propias NIIF para PYMES, basados en un criterio de los ingresos de sus actividades económicas - financieras, activos u otros factores que generen. En ocasiones, las PYMES originan estados financieros exclusivos para la toma de decisiones de los propietarios - gerentes, que a su vez, sirven para la presentación de información a los entes fiscales u otros organismos del Estado. La sección 1 de las NIIF completa, expresa que las NIIF para Pymes deben ser utilizadas por las pequeñas y medianas empresas que no tienen la obligación de rendir cuentas y aquellas que publiquen sus estados financieros con propósito de información general para usuarios externos (International Accounting Standards Board- (IASB), 2017).

Por otra parte, (Delgado \& Chávez, 2018), indican que en el Ecuador este tipo de empresas representan la mayor fuerza económica del Estado, siendo generadoras de empleo y promotoras de transcendentales avances en la sociedad local, la fuente de financiamiento para las Pymes en el Ecuador ha sido mediante créditos bancarios o a través de recursos propios, no obstante, una gran parte de éstas no crecen y no llegan a tener mucho tiempo en el mercado, a consecuencia de que varias no cumplen con los requisitos de estándares institucionales financieros para acceder a créditos.

En este orden de ideas, la aplicación de las NIIF por primera vez en el Ecuador, tuvo grandes controversias en la parte contable y la presentación de la información ante la entidad fiscal por las diferencias de criterio; es ahí que la NIC 41 sirve como guía de aplicación para el registro contable, expresando de manera directa que dicho registro se relacione con la actividad agrícola destinada a la cosecha o recolección, de acuerdo a su ciclo de producción. La aplicación de la norma debe ser exclusiva para el territorio o predio agrícola que realice esta actividad, considerando además a la NIC 16 Propiedades, Planta y Equipo; la NIC 40 Propiedades de Inversión; y, la NIC 38 Activos Intangibles (IASB, 2010). Debido al objeto del estudio, se debe mencionar a la NIC 2 Inventarios, la cual tiene como objetivo fundamental, establecer el procedimiento contable que permita el reconocimiento de los costos como parte del activo, hasta ser reconocidos 
Ingrid Anabel Arámbulo-Carrión; Jose Alciviades Guzmán-Ávila; Verónica Paulina Moreno-Narváez

como ingresos en la venta de los mismos. Esta norma proporciona las directrices para la determinación de los costos, para en lo posterior ser reconocidos como gastos dentro del ejercicio contable, incluyendo también el deterioro o pérdida que disminuya su valor en libros al valor neto realizable.

La sección 13 de las NIIF para Pymes contempla el reconocimiento de los inventarios, así como también, aquellos bienes que están almacenados para su reventa, como la mercadería adquirida por minoristas, terrenos u otras propiedades de inversión, y los productos terminados o en cursos de fabricación (Coronel, Narváez, \& Erazo, 2019). Por otra parte, la NIC 38 Activos Intangibles es aquella que determina el procedimiento contable de los activos intangibles que no estén considerados en otra norma, direccionándola al reconocimiento de un activo intangible sí y sólo si, se cumplen criterios de la mencionada norma, así también, enumera como establecer el registro en libros, y la exigencia para revelar información señalada sobre los activos de acuerdo a su clasificación y según la utilización de las partidas tales como: el conocimiento intelectual, el diseño e implementación de nuevos sistemas, contratos, licencias, propiedad intelectual, marcas, patentes, derechos de autor, capacitaciones, entre otros. Una de las características de esta norma es el reconocimiento y contabilización de la plusvalía, la misma que tiene relevancia para futuras revalorizaciones en la combinación de nuevos negocios que a diferencia de otras normas, no tuvo modificaciones y es significativa en su emisión realizada en el año 2011 (International Accounting Standards Board-(IASB), 2005); al respecto, se identifican dos tipos de activos intangibles, en primer lugar los activos intangibles adquiridos como por ejemplo los contratos y el segundo aquellos generados de manera interna como es el caso de capacitaciones y los proyectos para el cuidado del medio ambiente (Blacio, Narváez, \& Erazo, 2020).

Como se puede apreciar de acuerdo a lo expuesto, existe un cuerpo normativo de las NIIF que puede ser examinado bajo una perspectiva ambiental, con la finalidad de monitorear los activos, pasivos y desembolsos ambientales. En este sentido, 
considerando que la contabilidad se caracteriza por el reconocimiento, medición y valoración de los hechos económicos, el registro obligatorio de operaciones relacionadas con el ambiente generará responsabilidad para todas las empresas.

\section{El reconocimiento de los costos ambientales como instrumento para la toma de decisiones}

La contabilidad ambiental es el análisis entre el crecimiento económico y la conservación del medioambiente; lo cual empieza en el control contable tras la contaminación como efecto de la actividad empresarial (López-Jara, 2019), la cual tiene un impacto significativo en los años 80 por el descubrimiento de los daños ocasionados en las actividades productivas. El propósito de la contabilidad ambiental para (Alvarado, Ponguillo, \& Carrera, 2016) es presentar estados financieros para integrar políticas económicas y ambientales encaminadas a mitigar los daños causados al ecosistema y a construir empresas sostenibles. Bajo este concepto, se puede sintetizar que la contabilidad ambiental identifica el daño causado y el registro de los mismos, para tomar decisiones en acción de prevención que impulsen la conservación del ecosistema, valiéndose de herramientas que sirvan para mitigar el impacto generado, detallando tres acontecimientos:

1. Primero los problemas ambientales son problemas generados por los negocios, debido a que estos se presentan por el desarrollo de las actividades propias de la empresa, que a su vez pueden ser medibles por los valores detallados en el estado de resultados;

2. Segundo al ser parte de la administración ambiental, y por el conocimiento de cada uno de los costos incurridos, debe recomendar políticas que promuevan la reducción de los mismos;

3. Y tercero la realidad en que se presenta la información contable. 
Ingrid Anabel Arámbulo-Carrión; Jose Alciviades Guzmán-Ávila; Verónica Paulina Moreno-Narváez

Para la presentación de los estados financieros se debe tener en consideración que las cuentas ambientales tienen su naturaleza específica detallada a continuación:

- Activos ambientales: se consideran activos ambientales a todos los bienes y derechos que poseen las empresas para la preservación del medio ambiente, entre otros: activo circulante, activo permanente, propiedad ambiental y medio ambiente diferido; las cuentas empiezan y aumentan su movimiento en el debe, disminuye y se cancela en el haber, por lo general su saldo es deudor.

- Pasivos ambientales: son aquellas obligaciones que tienen las empresas por concepto de daños causados al medio ambiente, por lo general este grupo de cuentas es de difícil cuantificación debido que no se puede determinar el momento exacto de su ocurrencia; las cuentas empiezan y aumentan su movimiento en el haber, disminuyen y se cancelan en el debe, de manera habitual su saldo es acreedor.

- Patrimonio: se considera a las reservas que se realizan para afrontar episodios de contaminación severa que ocasionan las empresas, en este conjunto de cuentas están también las utilidades; los movimientos serán igual que las cuentas del pasivo.

- Ingresos medioambientales: es el aumento monetario que tiene relación directa con las actividades de conservación medioambiental, obtenidos por la reducción de los gastos incurridos, que se convierten en ahorros por su eficiente gestión que tienen naturaleza acreedora dentro del estado de resultados.

- Costos y gastos ambientales: conforma el valor monetario que se asigna a los efectos negativos de una actividad de producción que realizan las empresas para la sociedad; son cuentas que empiezan y aumentan en el debe, disminuyen y se cancelan en el haber, su saldo es deudor.

Teniendo en consideración que para la toma de decisiones no solo se debe analizar el balance general sino también el estado de resultados (Martínez, et al., 2020), y 
Ingrid Anabel Arámbulo-Carrión; Jose Alciviades Guzmán-Ávila; Verónica Paulina Moreno-Narváez

asumiendo que la presente investigación está enfocada en los costos ambientales, se conceptuaran los mismos, detallando los tipos que la conforman:

Costos ambientales: los costos ambientales son el resultado de las prácticas realizadas por las empresas para la conservación del medioambiente, con el objetivo de evitar, reducir y corregir los daños causados; o en otros términos es el registro de la restauración que sacrificó el ecosistema expresado de manera monetaria. Estos costos ambientales adquieren su valoración según (Salazar \& Montoya, 2014) de acuerdo al método que se implemente en su reparación:

Tipos de costos para la reparación ambiental:

- Costos de precios de mercado: es la valoración mediante la negociación de la oferta o la demanda de los servicios o productos registrados en el mercado dinámico.

- Costo de la empresa: son los costos estipulados por las empresas, en los cuales asignan costos específicos para la protección de medioambiente.

- Costos de la salud: este método toma en consideración el riesgo que tiene la población por el daño causado.

- Costo de oportunidad: es el costo que se reconoce en función a las pérdidas económicas generadas por la conservación del medioambiente.

- Costo de daño evitado: este método consiste en la apreciación implícita de los usuarios que eviten el daño causado en valores económicos.

- Costo de reparación: está enfocado en inversiones que ayuden a la reparación del recurso natural, en otro sitio diferente al inicial, como resultado de los daños.

En este sentido, (Panario, 2020) menciona que un plan de cuentas debe estructurarse de acuerdo a la ley y a los requerimientos de la empresa, debido a que es una guía importante del sistema contable que orienta cada uno de los registros y operaciones que se manejan de forma cotidiana. Las cuentas ambientales que se reflejan en un plan de cuentas serán las que están involucradas en la relación empresa-medio ambiente, conforme se evidencia en la figura 1: 


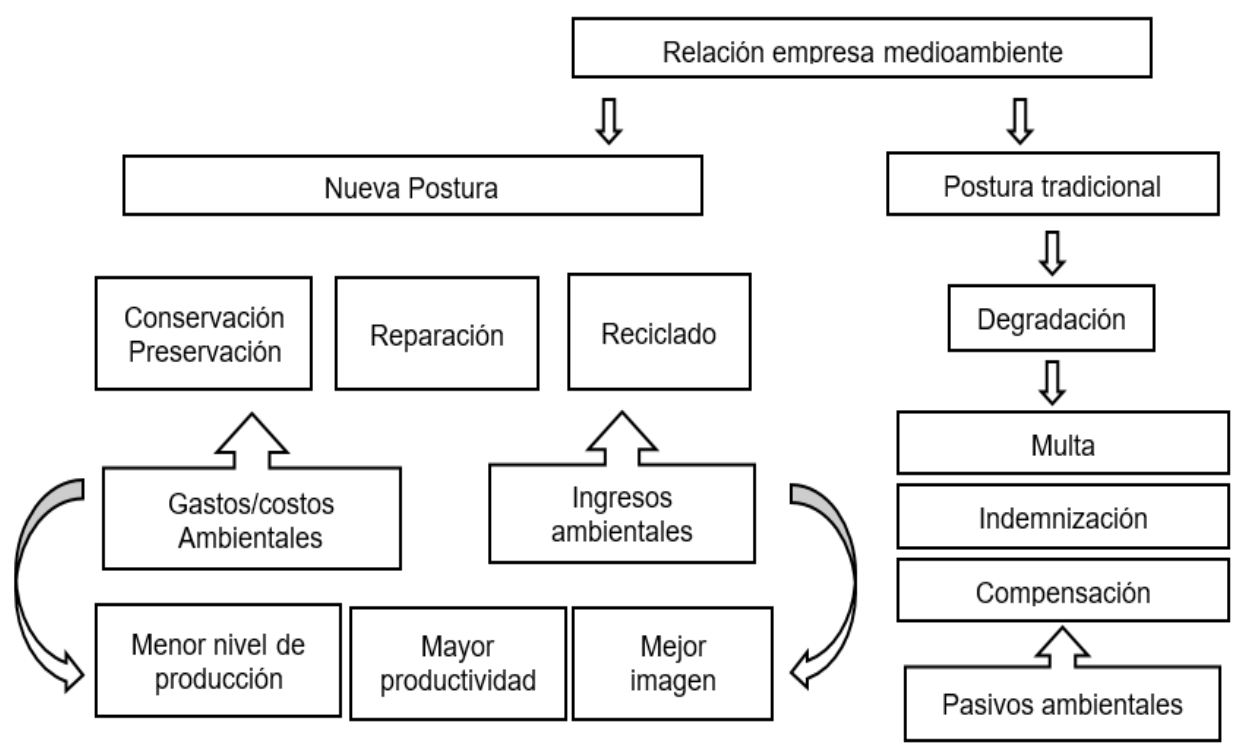

Figura 1. Relación empresa-medioambiente.

Fuente: Panario (2020).

\section{Los costos en el sistema de gestión ambientales para la toma de decisiones}

El tratamiento de los costos ambientales se lo debe realizar a través de un sistema que contenga políticas contables las cuales permitan a las empresas tener una guía en su desarrollo y aplicación; además de catalogar aquellas cuentas de naturaleza ambiental y de más indicadores que otorgan la medición y evaluación de la gestión ambiental en la toma de decisiones. Este conjunto de cuentas posibilita realizar reportes o informes ambientales como herramientas de orientación, prevención y registro de desarrollos ambientales que a su vez sirven para la aplicación de otras actividades. Se puede ampliar su presentación en el estado de resultados y el balance general, brindando a las Pymes una rápida identificación de sus costos y la confiabilidad al momento de tomar una decisión en la empresa.

Al mismo tiempo, para (Álvarez, Galaviz, \& Castro, 2019) un sistema de costos ambientales puede ser definido como el conjunto de procedimientos que según las leyes y normativa actual vigente, estén direccionadas a prevenir, reducir, reparar o conservar 
Ingrid Anabel Arámbulo-Carrión; Jose Alciviades Guzmán-Ávila; Verónica Paulina Moreno-Narváez

los recursos naturales en el medioambiente, en cada una de las etapas del proceso productivo. Por su parte, (Linares \& Suárez, 2017) consideran al sistema de costos ambientales como la evaluación de la gestión de cada uno de los costos, que permite el diseño e implementación de los procesos que prevengan la contaminación del medioambiente, haciendo uso del análisis del ciclo de vida de los costos identificados. La implementación de un sistema de costos ambientales en una empresa es considerado de vital importancia en todo el proceso productivo, tanto así que, su definición tiene diferentes conceptualizaciones al momento de desarrollarlo, sin embargo, persiguen el mismo objetivo, que es el cuidado del medio ambiente y la disponibilidad de la información para la toma de decisiones, tal como lo expresa los autores (Nakagoshi \& Saldaña, 2015) quienes afirman que un sistema de costos ambientales no solo sirve para el cálculo respectivo de los mismos, sino que además proporciona información útil para procesos administrativos en la toma de decisiones.

A partir de lo expuesto, se presenta un modelo de sistema de costos ambientales que se origina con la implementación de acciones preventivas o correctivas por parte de la empresa (De la Rosa, 2020) otorgando un tratamiento general para el cuidado del medio ambiente, a continuación se identifican procesos que tienen connotación ambiental (ver figura 2) y que forman parte del costo de producción, que en la actualidad no se reconocen y diferencian para el mejor control: 
Concepto medioambiental generado

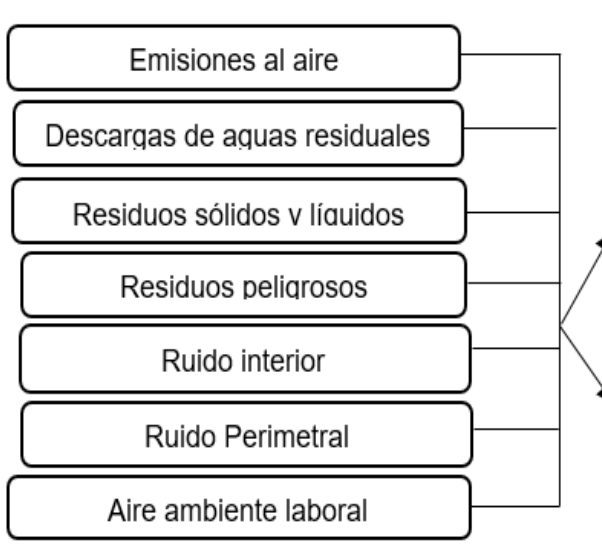

Factores claves de intervención

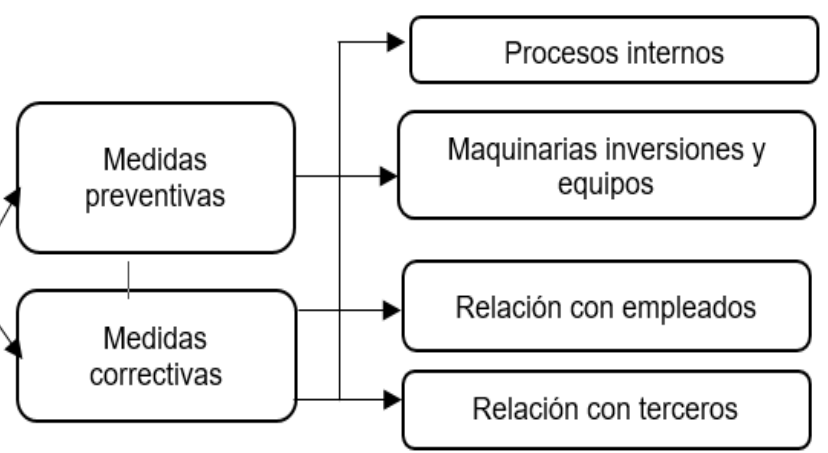

Figura 2. Modelo de costos ambientales.

Fuente: De la Rosa (2020).

Para el registro de las mencionadas acciones, es necesario que el sistema contable se encuentre actualizado (Lindao, Narváez, \& Erazo, 2019), para que refleje la situación actual de la empresa y puedan realizar provisiones necesarias por reparación y desmantelamiento del ambiente (Fierro \& Fierro, 2015); en este sentido, la toma de decisiones que ejecuten los propietarios y gerentes de las empresas debe mostrar una solución ante diversas situaciones que requieran atención urgente como manifiestan (Herrera \& Betancourt, 2016), para ello se realiza un análisis del problema con los siguientes componentes: elemento controlable e incontrolable, consecuencia de la posible decisión, limitaciones ambientales y estructurales, y correlación dinámica entre todos los componentes para implementar la decisión que solucione el inconveniente y que cumpla con los objetivos institucionales.

En la literatura especializada se evidencia varios métodos que ayudan a la toma de decisiones que dependen del momento y las cuestiones a definir, entre los más utilizados resaltan: la evaluación pros y contras que consiste en un diagnóstico del equilibrio entre 
Ingrid Anabel Arámbulo-Carrión; Jose Alciviades Guzmán-Ávila; Verónica Paulina Moreno-Narváez

beneficio y perjuicio, matriz FODA que realiza una gráfica de las fortalezas, oportunidades, debilidades y amenazas de la posible toma de decisiones, matriz océano azul que plantea las siguientes preguntas ¿Qué eliminar?, ¿Qué reducir?, ¿Qué incrementar? y ¿Qué crear?, diagrama de Ishikawa conocido como "espina de pescado" el cual consiste en ubicar el problema de manera vertical, como si fuera la columna vertebral de un pez y de ella se refleja las espinas que serían las causas de la situación a definir, mapas mentales que es un método muy potente que radica en colocar el problema en el centro del cual nace ramificaciones que serían las causas y por último el modelo de Kepner y Tregoe que es un esquema que pone de relieve las características principales de cada opción. Los métodos mencionados aportan a planificar y organizar los recursos y actividades de la empresa para gestionar el funcionamiento de la misma con el fin de cumplir los objetivos institucionales.

\section{MÉTODO}

La sostenibilidad medioambiental motiva al estudio sobre el impacto causado por el proceso productivo del sector agrícola en los ecosistemas, por lo tanto, el presente artículo se enmarcó en un diseño no experimental descriptivo considerando que no se manipuló de manera deliberada las variables en estudio, lo que permitió conocer los factores ambientales y su reconocimiento contable en el estado de resultados, es así que, se ejecutó un análisis del tratamiento contable de los costos ambientales y su relación con la toma de decisiones de la empresa, su finalidad fue de tipo transversal considerando que los datos fueron tomados en un solo periodo de tiempo.

Los métodos empleados fueron el analítico-deductivo, el cual se basó en investigar los costos ambientales que no son reconocidos en el proceso productivo, para así deducir un tratamiento contable para las Pymes bananeras; la técnica utilizada fue la encuesta y como instrumento de recolección de información el cuestionario el cual se conformó por 15 preguntas que se enfocaron en el área de gerencia, contabilidad, y producción, 
Ingrid Anabel Arámbulo-Carrión; Jose Alciviades Guzmán-Ávila; Verónica Paulina Moreno-Narváez

aplicadas mediante un censo. Para el bosquejo del instrumento se utilizó la escala de Likert y la confiabilidad del mismo se midió a través del estadígrafo Alfa de Cronbach que dio como resultado un coeficiente de 0,72 . El universo estuvo conformado por 110 Pymes bananeras del cantón Machala información que fue proporcionada por el MAGAP, el tipo de muestreo fue no probabilístico por conveniencia considerando como criterio de selección a bananeras con promedio de producción de 10 hectáreas, por tanto, la muestra ascendió a 60 bananeras.

\section{RESULTADOS}

La incorrecta aplicación del registro contable de los costos de las bananeras del cantón Machala evidenció un desnivel en las siguientes actividades que se detallan en la figura 3:

\begin{tabular}{|c|c|c|c|c|c|}
\hline \multirow{3}{*}{ 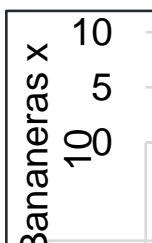 } & \multicolumn{5}{|c|}{ Resultados de encuesta } \\
\hline & & -1 & - & -1 & $\square$ \\
\hline & $\begin{array}{c}\text { Normativa } \\
\text { Interna }\end{array}$ & $\begin{array}{l}\text { Sistema de } \\
\text { costos }\end{array}$ & $\begin{array}{l}\text { Control de } \\
\text { gastos }\end{array}$ & Capacitaciones & $\begin{array}{l}\text { Toma de } \\
\text { decisiones }\end{array}$ \\
\hline$\stackrel{\sim}{\infty} \mathrm{Si}$ & 0,7 & 1,6 & 4,5 & 1,8 & 2,6 \\
\hline$\square$ No & 5,3 & 4,4 & 1,5 & 4,2 & 3,4 \\
\hline
\end{tabular}

Figura 3. Resultados de la encuesta.

Normativa interna: el personal que labora en las distintas áreas sujetas a la aplicación de la encuesta no tienen conocimiento de los procesos y cuidado del medioambiente, por la carencia de políticas y reglamentos internos acorde a la normativa vigente (capítulos II, III, IV y IX del Reglamento Interministerial para los sistemas aplicados en las bananeras emitido por el Ministerio del Ambiente, Ministerio de Salud Pública, Ministerio de Agricultura, Ganadería Acuacultura y Pesca, y la Dirección Nacional de Aviación Civil), por lo tanto, no tienen definido de manera técnica cada uno de los procesos a seguir en la producción. 
Ingrid Anabel Arámbulo-Carrión; Jose Alciviades Guzmán-Ávila; Verónica Paulina Moreno-Narváez

Sistema de costos ambientales: el $73,33 \%$ de los encuestados afirman que tanto los propietarios y el personal de las empresas no tienen conocimiento técnico del tratamiento de los costos ambientales, reflejando la importancia de implementar un sistema que les permita mejorar la clasificación y la asignación de cuentas contables que conlleve a la eficiente toma de decisiones.

Control de gastos ambientales: los costos ambientales se registran de manera directa en el estado de resultados, lo que impide tener un control eficiente de los costos incurridos, obteniendo como resultado que el $75 \%$ de los encuestados afirman que sus empresas registran los costos de producción en una sola cuenta sin clasificarlos por actividades, mientras que, el $39 \%$ restante proporcionó una respuesta positiva.

Capacitaciones: las empresas no capacitan al personal sobre los impactos ambientales que podrían ocasionar el mal manejo de los químicos y sustancias contaminantes que con el pasar de los años los llegaría a perjudicar de manera catastrófica en su salud y a las familias que habitan en sectores aledaños a las plantaciones de banano.

Toma de decisiones: el 56,67\% de encuestados señalan que las decisiones tomadas en la empresa con relación a temas ambientales se lo hace de forma empírica, debido a que en el estado de resultado no se reflejan de forma detallada las cuentas contables de connotación ambiental; esta práctica carente de fundamento técnico, afecta, no solo a la presentación de la información en los estados financieros, sino a las decisiones que se tomen entorno a la preservación del medio ambiente. 
Ingrid Anabel Arámbulo-Carrión; Jose Alciviades Guzmán-Ávila; Verónica Paulina Moreno-Narváez

Una vez que se han determinado las falencias que están afectando la continuidad de los procesos dentro de la empresa, es necesario tomar los correctivos pertinentes direccionados de forma explícita, en aquellos que tienen relación directa con los costos ambientales que servirán para una correcta toma de decisiones.

\section{PROPUESTA}

Con base en los resultados obtenidos se propone un sistema de costos ambientales que permita a las bananeras del cantón Machala, establecer el tratamiento contable de los costos ambientales y su presentación en los estados financieros para que los productores tomen decisiones de forma acertada (ver figura 4).

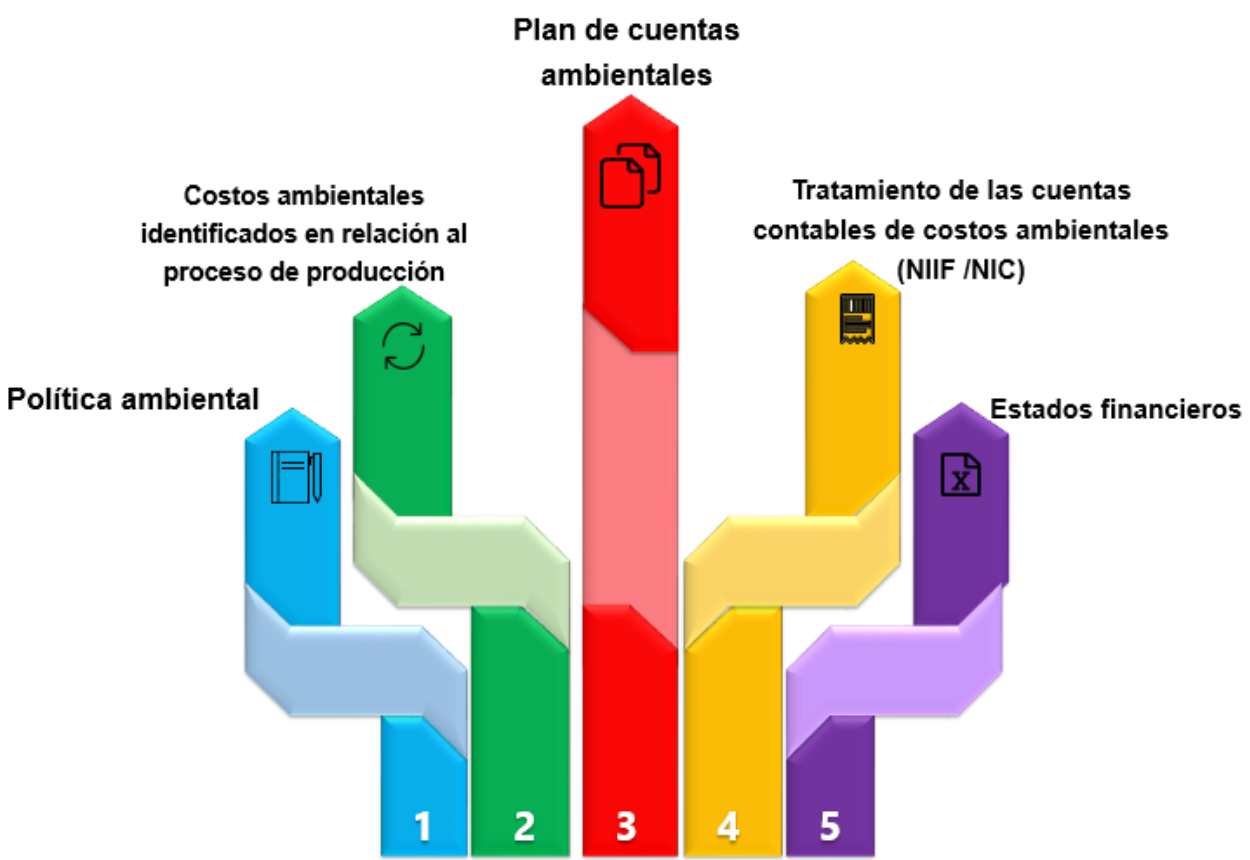

Figura 4. Sistema de costos ambientales. 
Ingrid Anabel Arámbulo-Carrión; Jose Alciviades Guzmán-Ávila; Verónica Paulina Moreno-Narváez

\section{Etapa 1: política ambiental}

Las siguientes políticas ambientales servirán para mejorar cada uno de los procesos ambientales en las bananeras, su tratamiento contable y presentación en los estados financieros:

- Realizar antes de la ejecución de un proyecto bananero los estudios de impacto ambiental y sus consecuencias negativas al ecosistema.

- Contener un plan interno de acción ambiental para la remediación de cada impacto o potencial impacto identificado.

- Poseer un inventario de productos contaminantes con todas las precauciones del caso, para evitar derrames que perjudiquen no solo al medioambiente, sino también la personal que los manipula.

- Construir un plan de cuentas específico que contenga directrices ambientales, de tal manera que los costos ambientales sean identificables.

- Realizar el tratamiento contable para los costos identificados dentro del proceso de producción.

- Realizar un control financiero de resultados, fundado en la eficiencia, la economía, la equidad y la valoración de los costos ambientales.

- Analizar los costos ambientales al terminar cada etapa de producción para conocer los valores monetarios incurridos con el fin de tomar decisiones de forma oportuna.

\section{Etapa 2: costos ambientales identificados con relación al proceso de producción}

El proceso de cultivo de banano exige ciertas características especiales de suelo y climatología, entre otras: ecología del banano, precipitación de agua, brillo solar y suelos. Así como también, requiere de varias fases en la producción, tales como: preparación de terreno, construcción de drenaje, siembra, riego, deshije/desoje, control de enfermedades, fertilización, cosecha y post cosecha (ver figura 5). 


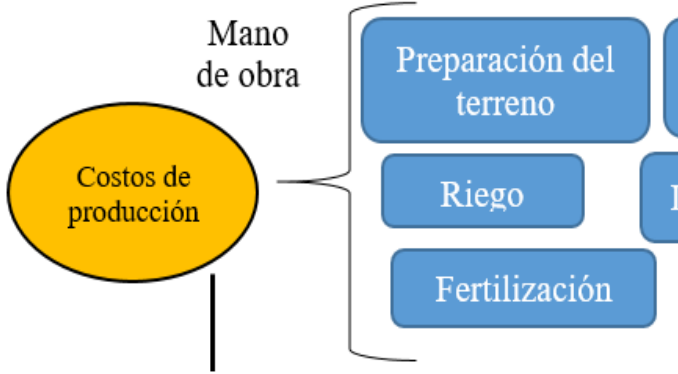

Costos directos e indirectos

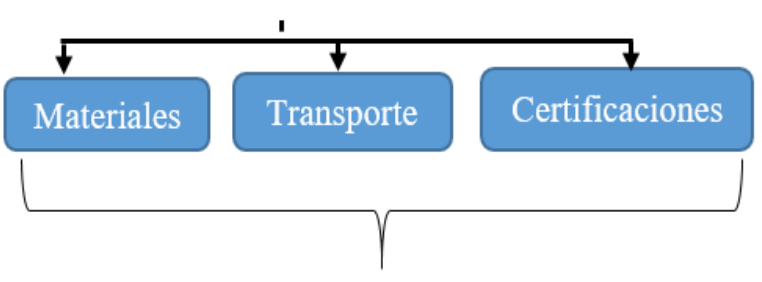

Instituciones de asistencia técnica. Apoyo a la producción y medioambiente

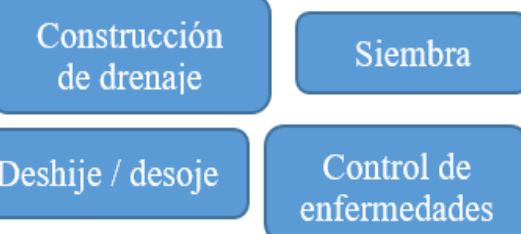

\section{Cosecha Post cosecha}

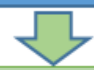

$\underline{\text { Reparación ambiental }}$

Costos de reparación

Costos de adquisición

Otros costos ambientales

Costos directos e indirectos

Figura 5. Identificación de costos ambientales en el proceso de producción.

\section{Etapa 3: plan de cuentas ambientales}

El plan de cuentas ambientales permitirá mejorar la percepción de la innovación en los registros de la contabilidad, en respuesta a los problemas que el impacto ambiental plantea en la industria bananera. A continuación, se considera un plan de cuentas que respalda de forma general a la terminología de la práctica contable internacional y se encuentra estructurado por 5 grupos de cuentas, con el siguiente orden: activo, pasivo, patrimonio, ingresos y costos. El plan incluye cuentas que pueden ser requeridas en situaciones actuales o predecibles en el futuro; los nombres de las cuentas reflejan la naturaleza de los hechos económicos que deben ser registrados en las mismas. En las cuentas se establecen rangos de códigos que posibilitan a las empresas bananeras habilitar las cuentas y subcuentas que requieran de acuerdo a sus características y especificidad (ver tabla 2). 
CIENCIAMATRIA

Revista Interdisciplinaria de Humanidades, Educación, Ciencia y Tecnología

Año VI. Vol. VI. N². Edición Especial II. 2020

Hecho el depósito de ley: pp201602FA4721

ISSN-L: 2542-3029; ISSN: 2610-802X

Universidad Nacional Experimental Francisco de Miranda (UNEFM). Santa Ana de Coro. Venezuela

Ingrid Anabel Arámbulo-Carrión; Jose Alciviades Guzmán-Ávila; Verónica Paulina Moreno-Narváez

\section{Tabla 2.}

Plan de cuentas ambientales.

\begin{tabular}{|c|c|}
\hline Código & Nombre de la Cuenta \\
\hline 1. & Activo \\
\hline 1.1. & Activo no corriente \\
\hline 1.1 .01 & Impreso ambiental \\
\hline 1.1 .02 & Reserva de cercas vivas \\
\hline 1.2. & Propiedad Ambiental \\
\hline 1.2 .01 & Filtros y equipos ambientales \\
\hline 1.1 .3 & Ambiente intangible \\
\hline 1.1.3.01 & Certificación ambiental Punto Verde \\
\hline 1.1.4. & Medio ambiente diferido \\
\hline 1.1.4.01 & Estudio de impacto ambiental \\
\hline 1.2. & Activo corriente \\
\hline 1.2.1. & Clientes \\
\hline 1.2.1.01 & Cuentas por cobrar Clientes ambientales \\
\hline 1.2.2. & Inventario \\
\hline 1.2.2.01 & Existencias de materias primas ambientales \\
\hline 1.2.2.02 & Existencias de productos en procesos ambientales \\
\hline 1.2.2.03 & Existencias de productos reciclados terminados \\
\hline 2. & Pasivo \\
\hline 2.1 .1 & Pasivo no corriente \\
\hline 2.1.1.01 & Proveedores de bienes ambientales \\
\hline 2.1.2. & Pasivo corriente \\
\hline 2.1.2.01 & Proveedores ambientales \\
\hline 2.1.2.02 & Compactación del suelo \\
\hline 2.1.2.03 & Contaminación del agua \\
\hline 2.1.2.04 & Provisión pasivos ambientales \\
\hline 3. & Patrimonio \\
\hline 3.1.1. & Reservas \\
\hline 3.1.1.01 & Reserva de Revalorización \\
\hline 3.1.1.02 & Ganancia o pérdida \\
\hline 4.1. & Ingresos \\
\hline 4.1.2. & Ingresos operacionales \\
\hline 4.1.2.01 & Venta por actividades operacionales \\
\hline 4.1.3. & Ingreso no operacional \\
\hline 4.1.3.01 & Venta de subproductos \\
\hline 4.1.3.02 & Ingreso por reciclaje \\
\hline 5.1. & Costos \\
\hline 5.1 .1 & Costos ambientales \\
\hline 5.1.1.01 & Costos de reciclaje de materiales \\
\hline 5.1 .1 .03 & Costos de preservación y recuperación ambiental \\
\hline 5.1.1.04 & Costos por fertilizantes y químicos ambientales \\
\hline 5.2.1.02 & Costos por suministros ecológicos \\
\hline 5.2.1.04 & Costos mantenimiento y equipamiento ambiental \\
\hline 5.2.1.05 & Costos reparación ambiental \\
\hline 5.2.1.06 & Costos por multa ambiental \\
\hline
\end{tabular}


Ingrid Anabel Arámbulo-Carrión; Jose Alciviades Guzmán-Ávila; Verónica Paulina Moreno-Narváez

\section{Etapa 4: tratamiento de las cuentas contables de costos ambientales (NIIF /NIC)}

A continuación, se describe el tratamiento contable de las distintas partidas una vez que las incidencias de los impactos medioambientales se tienen en consideración dentro de la contabilidad, a efectos de fortalecer el continuo perfeccionamiento de la información contable-financiera del sector bananero. Por consiguiente, se expone de forma explícita, un conjunto de asientos tipo que posibilitan el adecuado registro de las operaciones o hechos económicos que incluyen la interacción directa e indirecta de las empresas bananeras con el medio ambiente. Además, para homogeneizar puntos de referencia, se describen situaciones que incluyen partidas medioambientales que son de uso común en las operaciones de producción de banano en un ejercicio contable, de esta forma se procura la presentación práctica de los asientos tipo, con un lenguaje común y comprensible para los propietarios, directivos, personal contable y funcionarios de las bananeras (ver tablas 3-13).

Tabla 3.

Asiento inicial para la provisión del costo ambiental.

\begin{tabular}{|c|c|c|c|}
\hline \multicolumn{4}{|c|}{ Asiento de diario costo ambiental } \\
\hline Fecha & Detalle & Debe & Haber \\
\hline 01-oct-19 & $\begin{array}{l}\text { Provisión pasivos ambientales } \\
\text { Proveedores ambientales } \\
\text { P/R Provisión por reparación ambiental }\end{array}$ & $x x x x x$ & $x x x x x$ \\
\hline 05-oct-19 & $\begin{array}{l}\text { Costo de reparación ambiental } \\
\text { Provisión de pasivos ambientales } \\
\text { P/R La provisión de pasivos ambientales antes del trabajo }\end{array}$ & $\mathbf{X X X X X}$ & $x x x x x$ \\
\hline 05-oct-19 & $\begin{array}{l}\text { Proveedor ambiental } \\
\quad \text { Bancos } \\
\text { P/R pago del } 50 \% \text { por la reparación ambiental }\end{array}$ & $\mathbf{X X X X X}$ & $x X X X X$ \\
\hline 03-nov-19 & $\begin{array}{l}\text { Costo por reparación ambiental } \\
\text { Provisión pasivos ambientales } \\
\text { P/R Saldar la provisión de pasivos ambientales terminado el trabajo }\end{array}$ & $\mathbf{X X X X X}$ & $x X X X X$ \\
\hline 03-nov-19 & $\begin{array}{l}\text { Proveedores pasivo ambiental } \\
\text { Bancos } \\
\text { P/R pago final por la reparación ambiental }\end{array}$ & $x X X X X$ & $x x x x x$ \\
\hline
\end{tabular}


CIENCIAMATRIA

Revista Interdisciplinaria de Humanidades, Educación, Ciencia y Tecnología

Año VI. Vol. VI. N². Edición Especial II. 2020

Hecho el depósito de ley: pp201602FA4721

ISSN-L: 2542-3029; ISSN: 2610-802X

Universidad Nacional Experimental Francisco de Miranda (UNEFM). Santa Ana de Coro. Venezuela

Ingrid Anabel Arámbulo-Carrión; Jose Alciviades Guzmán-Ávila; Verónica Paulina Moreno-Narváez

Tabla 4.

Asiento contable del costo ambiental en los estados financieros.

\begin{tabular}{|c|c|c|c|}
\hline \multicolumn{4}{|c|}{ Contabilidad General } \\
\hline Fecha & Detalle & Debe & Haber \\
\hline 05-oct-19 & $\begin{array}{l}\text { Costo por reparación ambiental } \\
\text { Bancos } \\
\text { Documentos por pagar } \\
\text { P/R pago del } 50 \% \text { por la reparación ambiental }\end{array}$ & $\mathbf{x x x x x}$ & $\begin{array}{l}X X X X X \\
X X X X X\end{array}$ \\
\hline 03-nov-19 & $\begin{array}{l}\text { Documentos por pagar } \\
\quad \text { Bancos } \\
\text { P/R Desembolso total realizado por pago de reparación }\end{array}$ & $x x x x x$ & $x x x x x$ \\
\hline
\end{tabular}

De esta manera quedan establecidas cada una de las transacciones que se realizan al inicio del proceso de ejecución de la reparación ambiental y la presentación de las cuentas en los estados financieros; no obstante, existen más gastos que se presentarán en el transcurso de la ejecución de la reparación.

En el caso de obtener multas emitidas por el ente regulador se muestra la correcta aplicación de los asientos contables con las cuentas que intervienen en dicha transacción (ver tabla 5 y 6 ).

Tabla 5.

Asiento de diario por multa ambiental.

\begin{tabular}{|c|c|c|c|}
\hline \multicolumn{4}{|c|}{ Asiento de diario costo ambiental } \\
\hline Fecha & Detalle & Debe & Haber \\
\hline 04-Nov-19 & $\begin{array}{l}\text { Provisión de multa costos ambientales } \\
\text { Proveedores ambientales } \\
\text { P/R Provisión por multa ambiental }\end{array}$ & $\mathbf{X X X X X}$ & $x X X X X$ \\
\hline 05-Nov-19 & $\begin{array}{l}\text { Costo multa ambiental } \\
\text { Provisión de multa gastos ambientales } \\
\text { P/R El gasto por multas ambientales }\end{array}$ & $\operatorname{xxxxx}$ & $x x x x x$ \\
\hline 05-Nov-19 & $\begin{array}{l}\text { Proveedores ambientales } \\
\text { Bancos } \\
\text { P/R pago por multa ambiental }\end{array}$ & $x X X X X$ & $x x x x x$ \\
\hline
\end{tabular}


CIENCIAMATRIA

Revista Interdisciplinaria de Humanidades, Educación, Ciencia y Tecnología

Año VI. Vol. VI. N². Edición Especial II. 2020

Hecho el depósito de ley: pp201602FA4721

ISSN-L: 2542-3029; ISSN: 2610-802X

Universidad Nacional Experimental Francisco de Miranda (UNEFM). Santa Ana de Coro. Venezuela

Ingrid Anabel Arámbulo-Carrión; Jose Alciviades Guzmán-Ávila; Verónica Paulina Moreno-Narváez

Tabla 6.

Asiento contable por pago de multa ambiental.

\begin{tabular}{|c|c|c|c|}
\hline \multicolumn{4}{|c|}{ Contabilidad General } \\
\hline Fecha & Detalle & Debe & Haber \\
\hline 05-nov-19 & $\begin{array}{l}\text { Costo multas ambientales } \\
\text { Bancos } \\
\text { P/R Pago de multa al ente de contro }\end{array}$ & $x x x x x$ & $x x x x x$ \\
\hline
\end{tabular}

$\overline{\text { En el caso de venta de materiales reciclados se reclasificarán las cuentas, a continuación, }}$ se presenta el asiento contable sin cuentas ambientales y el segundo registro clasificando el ingreso por venta de materiales reciclables que son actividades operacionales que tienen las bananeras (ver tabla 7 y 8 ).

Tabla 7.

Asiento por venta de productos reciclados.

\begin{tabular}{cccc}
\hline & & Asiento Ingreso ambiental & \\
\hline Fecha & Detalle & Debe & Haber \\
\hline 14 -nov-19 & Bancos & Xxxxx & Xxxxx \\
& Otros ingresos & & \\
& P/R Ingresos por venta de material reciclable & & \\
\hline \hline
\end{tabular}

Tabla 8.

Asiento por ingresos de la venta de materiales reciclables.

\begin{tabular}{cccc}
\hline & & Contabilidad General & Debe \\
\hline Fecha & \multicolumn{1}{c}{ Detalle } & Haber \\
\hline 14-nov-19 & Bancos & Ingresos por reciclaje \\
& P/R Ingresos por venta de material reciclable & & xxxxx \\
& & \\
\hline \hline
\end{tabular}

Además, como parte de apoyo al medio ambiente la empresa adquiere una nueva política para la conservación del ecosistema y enuncia que se optimice la utilización de las resmas de papel y su reutilización, determinando también que estos se detallen en la estructura de los estados financieros, conforme se identifica en las tablas 9 y 10. 
CIENCIAMATRIA

Revista Interdisciplinaria de Humanidades, Educación, Ciencia y Tecnología

Año VI. Vol. VI. N². Edición Especial II. 2020

Hecho el depósito de ley: pp201602FA4721

ISSN-L: 2542-3029; ISSN: 2610-802X

Universidad Nacional Experimental Francisco de Miranda (UNEFM). Santa Ana de Coro. Venezuela

Ingrid Anabel Arámbulo-Carrión; Jose Alciviades Guzmán-Ávila; Verónica Paulina Moreno-Narváez

Tabla 9.

Asiento por compra de resma de papel.

\begin{tabular}{cccc}
\hline & & Asiento de gasto ambiental & \\
\hline Fecha & Detalle & Debe & Haber \\
\hline 15-nov-19 & Costo de suministros & Xxxxx & \\
& Banco & & \\
& P/R Gasto de resmas de papel reciclable & \\
\hline \hline
\end{tabular}

Tabla 10.

Asiento por costo de suministros ecológicos.

\begin{tabular}{cccc}
\hline \multicolumn{1}{c}{ Contabilidad General } & Debe & Haber \\
\hline Fecha & \multicolumn{1}{c}{ Detalle } & xxxxx & \\
\hline 15-nov-19 & $\begin{array}{c}\text { Costo de suministros ecológicos } \\
\text { Banco }\end{array}$ & & xxxxx \\
& P/R Gasto de resmas de papel reciclable & \\
\hline \hline
\end{tabular}

De igual manera, se presenta la reclasificación de la estructura de las cuentas contables de las adquisiciones de agroquímicos, conforme el asiento tradicional y el segundo asiento con la cuenta ambiental específica para esta compra, ver tablas 11 y 12 .

Tabla 11.

Asiento por compra de químicos y fertilizantes para conservar el medioambiente.

\begin{tabular}{cccc}
\hline \multicolumn{3}{c}{ Asiento de costo ambiental } & \\
\hline Fecha & Detalle & Debe & Haber \\
\hline 15 -nov-19 & $\begin{array}{c}\text { Costos por compra de agroquímicos } \\
\text { Banco }\end{array}$ & xxxxx & \\
& & & \\
P/R Costo de agroquímicos. & & \\
\hline \hline
\end{tabular}

Tabla 12.

Asiento adquisición de agroquímicos ecológicos.

\begin{tabular}{cccc}
\hline \multicolumn{3}{c}{ Asiento de costo ambiental } & \\
\hline Fecha & Detalle & Debe & Haber \\
\hline 15-nov-19 & $\begin{array}{c}\text { Costos por fertilizantes y químicos ambientales } \\
\text { Banco }\end{array}$ & xxxxx & \\
& & \\
P/R Costo de agroquímicos. & & \\
\hline \hline
\end{tabular}


Ingrid Anabel Arámbulo-Carrión; Jose Alciviades Guzmán-Ávila; Verónica Paulina Moreno-Narváez

Por los cambios en la estructura contable y los procesos de mejoras en el medioambiente, es necesario la adquisición de una marca ecológica proyectada al incremento de las ventas, denominada certificación punto verde considerada importante en el mercado internacional (ver tabla 13).

Tabla 13.

Certificación punto verde.

\begin{tabular}{clcc}
\hline \multicolumn{1}{c}{ Contabilidad General } & & Debe & Haber \\
\hline Fecha & \multicolumn{1}{c}{ Detalle } & xxxxx & \\
\hline 30-Nov-19 & Derechos de marca & xxxxx & xxxxx \\
& Certificación ambiental punto verde & & \\
& Reserva de revalorización (patrimonio neto) & & \\
P/R Registro de valorización de la marca & & \\
\hline \hline
\end{tabular}

\section{Etapa 5: estados financieros}

Con la finalidad de incorporar conceptos ambientales en los estados financieros y en las notas a los estados financieros es necesario contextualizar las normas contables del medio ambiente, de tal forma que se exprese de manera clara los rubros de información medioambiental utilizados. En consecuencia, se procede a realizar un comparativo entre los estados financieros tradicionales y los ambientales, en el primero se observa un balance general y un estado de resultados sin connotación ambiental que utilizan las bananeras de forma habitual; mientras que, en el segundo se presenta un balance general con el respectivo estado de resultado utilizando la clasificación de las cuentas ambientales de acuerdo a la reestructuración del plan de cuentas (ver tablas 14-17). 
CIENCIAMATRIA

Revista Interdisciplinaria de Humanidades, Educación, Ciencia y Tecnología

Año VI. Vol. VI. N². Edición Especial II. 2020

Hecho el depósito de ley: pp201602FA4721

ISSN-L: 2542-3029; ISSN: 2610-802X

Universidad Nacional Experimental Francisco de Miranda (UNEFM). Santa Ana de Coro. Venezuela

Ingrid Anabel Arámbulo-Carrión; Jose Alciviades Guzmán-Ávila; Verónica Paulina Moreno-Narváez

Tabla 14.

Balance General (tradicional).

\begin{tabular}{|c|c|c|}
\hline \multicolumn{3}{|c|}{ Balance General } \\
\hline Activo & & $x X X X X$ \\
\hline Bancos & $x x x x x$ & \\
\hline Equipos de computación & $x x x x x$ & \\
\hline Maquinaria y equipo & $\mathrm{XXXXX}$ & \\
\hline Terreno & $\mathrm{xXXXX}$ & \\
\hline Edificio & $\mathrm{XXXXX}$ & \\
\hline Pasivo & & $x x x x x$ \\
\hline Cuentas por pagar & $x x x x x$ & \\
\hline Documentos por pagar & $x x x x x$ & \\
\hline Patrimonio & & $x x x x x$ \\
\hline Capital social & $x x x x x$ & \\
\hline Reserva & $x x x x x$ & \\
\hline Utilidad o pérdida del ejercicio & $x x x x x$ & \\
\hline
\end{tabular}

\section{Tabla 15.}

Estado de Resultados (tradicional).

\begin{tabular}{|c|c|c|}
\hline Estadc & & \\
\hline Ingresos & & $x x x x x$ \\
\hline Ventas & $\operatorname{xxxxx}$ & \\
\hline Otros ingresos & $\operatorname{xxxxx}$ & \\
\hline Costos & & $x x x x x$ \\
\hline Gastos por multas & $\operatorname{xxxxx}$ & \\
\hline Costos de suministros & $\operatorname{xxXXX}$ & \\
\hline Costos operacionales & $\operatorname{xxXXX}$ & \\
\hline Costos de reparación & $\operatorname{xxXXX}$ & \\
\hline Costos de fundas y envases & $\operatorname{XXXXX}$ & \\
\hline Utilidad del ejercicio & & $\mathbf{X X X X X}$ \\
\hline
\end{tabular}


CIENCIAMATRIA

Revista Interdisciplinaria de Humanidades, Educación, Ciencia y Tecnología

Año VI. Vol. VI. N². Edición Especial II. 2020

Hecho el depósito de ley: pp201602FA4721

ISSN-L: 2542-3029; ISSN: 2610-802X

Universidad Nacional Experimental Francisco de Miranda (UNEFM). Santa Ana de Coro. Venezuela

Ingrid Anabel Arámbulo-Carrión; Jose Alciviades Guzmán-Ávila; Verónica Paulina Moreno-Narváez

Tabla 16.

Balance General (ambiental).

Balance General

\begin{tabular}{|c|c|c|}
\hline & & 人А^А⿵ \\
\hline Activo & & \\
\hline Bancos & Xxxxx & \\
\hline Bancos cuenta ambiental & Xxxxx & \\
\hline Equipos de computación & Xxxxx & \\
\hline Maquinaria y equipo & Xxxxx & \\
\hline Terreno & Xxxxx & \\
\hline Edificio & Xxxxx & \\
\hline Derecho de marca & $\mathbf{X x x x x}$ & \\
\hline Licencia ambiental punto verde & $\mathbf{X x x x x}$ & \\
\hline Pasivo & & $X x x x x$ \\
\hline Documentos por pagar & $\operatorname{Xxxxx}$ & \\
\hline Pasivos ambientales & $\mathbf{X x x x x}$ & \\
\hline Patrimonio & & $X x x x x$ \\
\hline Capital social & Xxxxx & \\
\hline Reserva & XxxxX & \\
\hline Utilidad o pérdida del ejercicio & Xxxxx & \\
\hline Reserva de revalorización ambiental & $X x x x x$ & \\
\hline
\end{tabular}

Tabla 17.

Estado de Resultado (ambiental).

\begin{tabular}{lll}
\hline \multicolumn{1}{c}{ Estado de Resultados } & & \\
\hline Ingresos & & Xxxxx \\
\hline Ventas & $\mathbf{X x x x x}$ & \\
\hline Otros ingresos & $\mathbf{X x x x x}$ & \\
\hline Ingresos por reciclaje & & Xxxxx \\
\hline Costos & $\mathbf{X x x x x}$ & \\
\hline Costos por multas & $\mathbf{X x x x x}$ & \\
\hline Costos por multas ambientales & $\mathbf{X x x x x}$ & \\
\hline Costos de suministros & $\mathbf{X x x x x}$ & \\
\hline Costos suministros ecológicos & $\mathbf{X x x x x}$ & \\
\hline Costos operacionales & $\mathbf{X x x x x}$ & \\
\hline Costos de reparación & $\mathbf{X x x x x}$ & \\
\hline Costos de reparación - ambiental & $\mathbf{X x x x x}$ & \\
\hline Costos de fundas y envases & & Xxxxx \\
\hline Utilidad del ejercicio & & \\
\hline
\end{tabular}


Ingrid Anabel Arámbulo-Carrión; Jose Alciviades Guzmán-Ávila; Verónica Paulina Moreno-Narváez

Notas a los estados financieros: en las notas conexas se deberá especificar las operaciones contabilizadas que se encuentren relacionadas con el medio ambiente, por ejemplo: las contingencias ambientales, por consiguiente, se revelarán los hechos económicos no son recurrentes y que tienen incidencia material en los estados financieros.

\section{CONCLUSIÓN}

La aplicación de las normas internacionales de información financiera tendrá un impacto significativo en la determinación de los costos ambientales por los lineamientos que en ella se definen, los cuales establecen los requisitos para el reconocimiento, medición, presentación e información a revelar sobre las transacciones y hechos económicos que afectan a la empresa y que se reflejan en los estados financieros.

La contabilidad ambiental permite conocer el superávit y déficit económico, en conjunto con la conservación del medioambiente; lo cual incide en el control contable tras la contaminación como efecto de la actividad empresarial, y los daños ocasionados por las actividades productivas, con el fin de valorar los fenómenos en materia económica. En este contexto, la identificación y clasificación de los costos ambientales en la etapa de producción permitirá evaluar y tomar decisiones apropiadas con relación al ahorro de costos, maximización de beneficios y disminución de riesgos económicos y ambientales. Una vez realizado el análisis comparativo de la información de los estados financieros en la propuesta, se pudo deducir que la aplicación de las NIIF para PYMES (tratamiento de los activos biológicos expresados en la NIC 41, reconocimiento de los activos para el cuidado del medio ambiente como parte integrante de la propiedad, planta y equipo de la empresa, establecido en la NIC 16, y el sistema de costos ambientales que conlleva la reestructuración del plan de cuentas, la presentación detallada y sistemática de los estados financieros), permitirá a los productores obtener información minuciosa de cada 
Ingrid Anabel Arámbulo-Carrión; Jose Alciviades Guzmán-Ávila; Verónica Paulina Moreno-Narváez

uno de los costos incurridos en el ámbito medioambiental, con lo cual, los gerentes o administradores, podrán tomar decisiones acertadas que ayuden no solo a la continuidad productiva de las empresas bananeras;

Sino también a la protección y conservación del medioambiente, además, la obtención de beneficios y privilegios que otorgan las empresas certificadoras a nivel nacional e internacional, con el aval de las entidades de control; que se obtienen por el cumplimiento de indicadores o parámetros establecidos por estos. Por último, basados en el concepto de la toma de decisiones, la aplicación de la misma le permitirá al productor bananero, identificar posibles riesgos, errores o incumplimiento de la normativa vigente.

\section{REFERENCIAS CONSULTADAS}

Alvarado, E., Ponguillo, K., \& Carrera, F. (2016). Reflexiones sobre la Contabilidad Ambiental. [Reflections on Environmental Accounting]. Revista Publicando, 3(7), 156-166.

Álvarez, R., Galaviz, B., \& Castro, J. (2019). Contabilidad Ambiental para la toma de decisiones: fundamentos y practicas. [Environmental Accounting for decision making: fundamentals and practices]. Identidad bolivariana, 3(1), 9-37.

Blacio, C., Narváez, I., \& Erazo, J. (2020). Normas internacionales de información financiera y reconocimiento contable de jugadores de fútbol en clubes deportivos. [International standards for financial information and accounting recognition of soccer players in sports clubs]. Revista Arbitrada Interdiciplinaria Koinonía, 5(10), 34-62. http://dx.doi.org/10.35381/r.k.v5i10.687

Coronel, J., Narváez, C., \& Erazo, J. (2019). Análisis del impacto financiero y tributario a partir de la valuación de inventarios bajo NIIF en la empresa VETNAR S.A. [Analysis of the financial and tax impact from the valuation of inventories under IFRS in the company]. Revista Arbitrada Interdisciplinaria Koinonía, 4(2), 56-83.. http://dx.doi.org/10.35381/r.k.v4i2.467

De la Rosa, M. (2020). Foro virtual de contabilidad ambiental y social. Obtenido de Foro virtual de contabilidad ambiental y social: https://url2.cl/RN6zp 
Ingrid Anabel Arámbulo-Carrión; Jose Alciviades Guzmán-Ávila; Verónica Paulina Moreno-Narváez

Delgado, D., \& Chávez, G. (2018). Las Pymes en el Ecuador y sus fuentes de financiamiento. [smes in Ecuador and their sources of financing]. Revista observatorio de la economía Latinoamericana. Obtenido de https://url2.cl/88x6t

Fierro, A., \& Fierro, F. (2015). Contabilidad de activos con enfoque NIIF para Pymes. [Asset accounting with an IFRS approach for smes]. Bogota: Ecoe ediciones. Obtenido de https://url2.cl/Uxyyl

Herrera, A., \& Betancourt, V. (2016). Razones financieras de liquidez en la gestion empresarial para toma de decisiones. QUIPUKAMAYOC, 151-160. Obtenido de https://n9.cl/isf7e

IASB. (2010). Internacional Accounting Standards Boards. [Normas Iternacionales de contabilidad]. Recuperado de https://url2.cl/ADwga

International Accounting Standards Board- (IASB). (2017). NIIF para Pymes, Norma Internacional de Información Financiera para Pequeñas y Medianas Entidades. Obtenido de https://n9.cl/9yzi

International Accounting Standards Board-(IASB). (2005). Norma Internacional de Contabilidad 41 Agricultura. Obtenido de https://url2.cl/78Uvw

Linares, M., \& Suárez, Y. (2017). Los costos ambientales: un análisis de la producción científica en el periodo 1977-2016 y una revisión de herramientas y teorías subyacentes. [Environmental costs: an analysis of scientific production in the period 1977-2016 and a review of the underlyin]. Criterio Libre 15 (27), 89-114.

Lindao, D., Narváez, I., \& Erazo, J. (2019). La contabilidad de gestión estratégica como herramienta multidisciplinar de planificación y control en la exportadora bananera Novamerc S.A. [Strategic management accounting as a multidisciplinary planning and control tool in the banana exporter Novamerc]. Revista Arbitrada Interdisciplinaria Koinonía, 4(2), 147-182.

López Jara, Ana Alexandra. (2019). La contabilidad de gestión ambiental como herramienta para generar sostenibilidad empresarial. Revista Investigación y Negocios, 12(19), 18-27. 
Ingrid Anabel Arámbulo-Carrión; Jose Alciviades Guzmán-Ávila; Verónica Paulina Moreno-Narváez

Martínez, I., Martínez, L., \& De León, I. (2020). La importancia del análisis de los estados financieros en la toma de decisiones. [The Importance of Financial Statement Analysis in Decision Making]. Obtenido de https://url2.cl/u2uW7

Nakagoshi, K., \& Saldaña, C. (2015). La evolucion de los sistemas de costos en un entorno economico cambiante. [The evolution of cost systems in a changing economic environment]. Vincula Tégica, 1097-1112. Obtenido de https://url2.cl/UAIFG

Panario, M. (2020). Cuentas ambientales: un plan de cuentas integrado ¿si o no?.[Environmental accounts: an integrated chart of accounts, yes or no?]. Obtenido de https://url2.cl/uH1gs

Ron, R., \& Sacoto, V. (2017). Las Pymes ecuatorianas: su impacto en el empleo como contribución del PIB Pymes al PIB total. [Ecuadorian smes: their impact on employment as a contribution of GDP smes to total GDP]. Revista Espacios. Obtenido de https://url2.cl/XuXDU

Salazar, W., \& Montoya, D. (2014). Los costos ambientales en la sostenibilidad empresarial. Propuesta para su valoración y revelación contable.[environmental costs in business sustainability Proposal for its valuation and accounting disclosure]. Contaduría Universidad de Antioquia. Obtenido de https://url2.cl/CCZHu

Yance, C., Solís, L., Burgos, I., \& Hermida, L. (2017). La importancia de la Pymes en el Ecuador. [The importance of SMEs in Ecuador]. Observatorio de la economía Latinoamericana. Obtenido de https://url2.cl/Y5glx 